\title{
Theory of Mind Increases Aesthetic Appreciation in Visual Arts
}

\author{
Marina Iosifyan* \\ St Mary's College, School of Divinity, University of St Andrews, South Street, \\ St Andrews, KY16 9JU, UK
}

Received 23 April 2020; accepted 18 October 2020

\begin{abstract}
Theory of mind is a cognitive ability that enables us to understand mental states of others, important in real-life communications as well as in aesthetic cognition. The present research investigated whether understanding intentions and emotions is related to aesthetic appreciation. Study 1 tested whether there is a link between aesthetic appreciation of cinematic films and attempts to understand the intentions and emotions of the artists and the film characters. It showed that a self-reported understanding of emotions and intentions is positively associated with aesthetic appreciation. Studies 2 and 4 investigated a causal relationship between the attempt to understand emotions and an aesthetic appreciation of artistic photos. Study 3 investigated an actual understanding of emotions and aesthetic appreciation of movie shots. The results show that when people evaluate the emotional state of the characters, they aesthetically appreciate artistic photos more, compared to when they evaluate nonmental characteristics of these photos (age of the characters, the colour of the photos). Moreover, better understanding of another's emotions is related to greater aesthetic appreciation.
\end{abstract}

\section{Keywords}

Theory of mind, understanding intentions, understanding emotions, aesthetic appreciation, cinema

\section{Introduction}

Theory of mind (sometimes called mentalizing and mind reading) is the ability to understand mental states of others. It is the key element of social cognition which enables people to understand and to predict the behaviours of others and to adequately respond to them. Theory of mind is also involved in

\footnotetext{
*To whom correspondence should be addressed. E-mail: marina.iosifyan@gmail.com
}

This is an open access article distributed under the terms of the CC BY 4.0 license. 
aesthetic cognition. Imagine someone reading or watching Shakespeare's Romeo and Juliet without theory of mind. It would be impossible to understand the culmination of the tragedy: Romeo commits suicide because he thinks that Juliet is dead, while the reader knows that she is alive (that is, Romeo is having a false belief about Juliet's death). It is theory of mind that enables us to understand the underlying intentions, beliefs and emotions of the fictional characters (Zunshine, 2003). Readers and literary scholars alike endlessly debate the intentions of complex imaginary characters, such as Hamlet, and the authors who create them (Charnes, 2007; Levin, 1994; Werstine, 1988).

Theory of mind consists of several dimensions. In the present study, linking theory of mind and aesthetic appreciation, we regard two of them: the actual understanding of another's emotions/intentions and the thinking about and trying to understand another's emotions/intentions (whether successful or not). Moreover, we evaluated both the attempt to understand the artist's mind and the attempt to understand the character's mind. If these dimensions of theory of mind play an important role in aesthetic cognition, it is possible that it also affects art appreciation and aesthetic judgement.

Psychologists develop models explaining how people perceive and aesthetically appreciate art (Leder et al., 2004; Parsons, 1987). These models take into account perceptual, emotional and cognitive processes. According to such models, cognitive processes, such as intention understanding, play an important role in aesthetic appreciation. Cognitive processes are one of the key characteristics of aesthetic experience (Marković, 2012). Parsons (1987), who developed a cognitive developmental account of aesthetic experience and appreciation, explained that people appreciate a single work of art differently because they understand it differently. It was theorized that theory of mind and imagination are some of the key components of aesthetic appreciation, because art often depicts fictious words and narratives (Bullot and Reber, 2013). Perceiving these imaginary worlds, people attribute beliefs and desires to fictional characters. Moreover, when perceiving a work of art, the viewers are aware that it was created intentionally by the artist and thus attempt to understand her intentions (Parsons, 1987; Pelowski and Akiba, 2011). The art creator's identity has an impact on aesthetic appreciation of this art. For example, beliefs about art authenticity increase aesthetic appreciation, even if the same picture is presented as authentic or copied (Wolz and Carbon, 2014). It is particularly interesting, because understanding of the artist's intentions might be more complex since he or she is not directly presented in the artwork, while the characters are.

Perception of the underlying intentionality of artworks thus might affect aesthetic appreciation of these artworks. Imagine perceiving aesthetic qualities of a pair of glasses, lying on the ground, and a pair of glasses, lying on the floor as an exhibit in an art gallery. While the object is the same, its perception 
will be different: a pair of glasses left on a floor at San Francisco's Museum of Modern Art were mistaken for art and admired by the visitors. A recent study by Margulis and colleagues showed that information about the author's intent had an impact on music and poetry perception (Margulis et al., 2017). The information about the intent of the author affected the enjoyment and movingness ratings of music and poetry.

Neuroimaging research also showed that intentionality is part of aesthetic appreciation (Höfel and Jacobsen, 2007). In their fMRI study, Kirk and colleagues presented artworks (paintings) to their subjects in a human-made context and a non-human-made context (computer-generated). They found higher activation in temporal poles in the human-made context as opposed to the non-human-made context (Kirk et al., 2009). Temporal poles are consistently activated in association with theory of mind (Gallagher and Frith, 2003).

In order to establish a link between the actual understanding of another's emotions/intentions and art appreciation, some empirical research is needed. There are some experimental studies which partially support the idea that actual understanding of another's mind and aesthetic cognition are linked. Perception of music is related to emotion understanding and impacts the aesthetic appreciation of this music (Weth et al., 2015). For instance, human characteristics (e.g., sadness) are often attributed to musical passages (Juslin and Lauk$\mathrm{ka}, 2003)$. Mar and colleagues found that lifetime exposure to fiction is positively associated with correctness of understanding of another's mind (Mar et al., 2006, 2009). Theory of mind in the study was measured by the 'Reading the mind in the eyes' test (RMET; Baron-Cohen et al., 2001) and the Interpersonal Recognition Test (Costanzo and Archer, 1989). In RMET the participants are asked to look at the photos of people's eyes and express their opinion on the feelings or thoughts of those people by choosing one answer out of four. In the Interpersonal Recognition Test participants are asked to watch several videos and answer the questions about the interrelations of people in these videos.

Mar and colleagues found a link between lifetime exposure to fiction and the ability to correctly understand the other's emotions/intentions, which was then indirectly confirmed during the experiment of Kidd and Castano (2013) which showed that engagement in fiction has a positive impact on mind-reading ability. Kidd and Castano asked participants to read literary fiction during the experiment with subsequent RMET testing. It was found that reading fiction (as opposed to non-fiction) improved their theory of mind. This finding was replicated in several studies where the participants was asked to read a fiction story (Black and Barnes, 2015a), or to watch a fiction TV-drama (Black and Barnes, 2015b) with further RMET testing. In all cases the participants' scores in RMET were improved as opposed to those of people who read nonfiction. Although there were some unsuccessful attempts to replicate these findings (Panero et al., 2016), a recent meta-analysis has confirmed that read- 
ing fiction had a small positive effect on theory of mind (Dodell-Feder and Tamir, 2018; Mumper and Gerrig, 2017).

If there is a link between perceiving art fiction and the understanding of the other's mind, it is possible that this understanding has an impact on the aesthetic appreciation of fiction. A better understanding of a character's intentions, emotions, beliefs and the intentions of the artist who created them, may result in higher appreciation of this fiction. It is possible that there is a direct link between correct emotion/intention understanding and art appreciation. But it is also possible that this link is mediated by several factors. For instance, correct understanding of the other's mind may enhance empathy and selfidentification with the characters, and these variables might affect aesthetic experience (Freedberg and Gallese, 2007). However, to our knowledge, the hypothesis that correct understanding of the other's mind is positively related to art appreciation was not yet empirically tested.

Along with actual understanding of the other's mind, a bigger engagement in the other's mind might play an important role in aesthetic appreciation. Thinking about the mind of others plays an important role in cinema: being engaged in fiction, people are aware of its artificial nature, and that the artist's intentions are behind the fiction (Pignocchi, 2015). Imagine you are watching Ang Lee's drama "Life of P $i$ ". This movie describes the story of a young man, $\mathrm{Pi}$, travelling on a ship. After a storm that wrecks the ship, Pi finds himself on a lifeboat in the company of another survivor - a Bengal tiger. Pi and the tiger travel on a boat together and try to survive throughout the entire movie. Watching this, you may unintentionally attribute mental characteristics to that tiger. E.g., you may think that the tiger has the intention to help Pi, or that the tiger empathizes with $\mathrm{Pi}$, or that the tiger resists eating $\mathrm{Pi}$, because the latter has saved him from being drowned. Finally, you may also think of the tiger as a metaphor of fear, animal instinct, grief, rage, fate or even death. Now, imagine you face the same situation of a man trapped in one space with a tiger in real life. Would you still attribute the abovementioned mental qualities to the tiger? Will you perceive the tiger as a metaphor? It is highly unlikely. Being engaged in any type of fiction, as opposed to facing a real-life situation, we are aware of its artificial nature. This does not mean, though, that people correctly understand the intentions behind the fiction. It only signifies that engaging in fiction people attempt to understand these intentions (Pignocchi, 2015), and attempting to understand these intentions might thus enhance the aesthetic appreciation of cinema.

Investigating the role of different dimensions of theory of mind in visual arts is particularly interesting, because visual perception is important for theory of mind development (Flavell, 2004). While visually monitoring others, it is easier for the observer to understand their beliefs, intentions and emotions. Levin et al. (2013) suppose that theory of mind is the basis of cinema percep- 
tion. They argue that filmmakers use different techniques to help viewers understand the minds of their characters via visual narratives.

The present study is an explorative study that investigates the relations between intention and emotion understanding in visual arts - cinema and photography. We test the hypothesis that correct understanding of another's mind in the cinema and aesthetic appreciation of this cinema are linked. We also test the hypothesis that trying to understand another's emotions/intentions in cinema results in higher aesthetic appreciation of this cinema.

\section{Study 1}

The first study aimed to test the hypothesis that there is a link between theory of mind and aesthetic appreciation of cinematic films. Participants watched feature films and evaluated them aesthetically. After the aesthetic evaluation, participants reported how well they understood the intentions and emotions of the characters and of the film director. It was expected that there is a link between subjective emotion/intention understanding and aesthetic appreciation of the films. That is, films with high rating of emotion/intention understanding are highly aesthetically appreciated, and vice versa.

\subsection{Method}

\subsubsection{Participants}

There were two rounds of data collection with several months inbetween. Initially, 28 volunteers recruited at the university campus participated in the study. After the study they were asked how they understood the study hypothesis. Two of them responded that it is expected that the more they understand the intentions of characters and film director, the more they like the film. Thus, these participants were excluded from data analysis. During the second round of data collection, four more participants were recruited. Finally, 30 volunteers between 19 and 36 years of age participated in the study $\left(M_{\text {age }}=22.67\right.$, $\mathrm{SD}=6.98$; six males). Informed consent was obtained from all volunteers, and the experiment was carried out in accordance with the Code of Ethics of the World Medical Association (Declaration of Helsinki). All studies described in this paper were approved by the Ethical Committee of the Institute of Psychology of the Russian Academy of Sciences.

\subsubsection{Materials}

Eight short feature films were used in the study ("One Fine Day" by Takeshi Kitano; "The Lady Bug" by Jane Campion; "Artaud Double Bill" by Atom Egoyan; "The Foundry" by Aki Kaurismaki; "Upsurge" by Olivier Assayas; "I 
Travelled 9000 km To Give It To You" by Wong Kar-Wai; "Awkward" by Elia Suleiman; "The Last Dating Show" by Bille August). The length of the films ranged from 2.57 to 3.51 minutes $(M=2.96, S D=.29)$. All short feature films were extracted from an anthology film, "To Each His Own Cinema" (2006), made by different film directors. These films were chosen because of their short length (2-3 minutes each), which makes in unnecessary to cut them. Moreover, choosing short movies from one anthology film allowed achieving a relative uniformity of stimuli (all films are made by world-leading film directors).

Aesthetic appreciation of the films was measured on a scale from 1 to 10 , rating how much he/she liked it (1, not at all; 10, very much) and the likelihood that she/he would go to the cinema to watch that film (1, not likely; 10, very likely). Emotion and intention understanding were measured using selfreport answering the following questions: (1) How well did you understand the intentions of the characters? (2) How well did you understand the feelings of the characters? (3) How well did you understand the intentions of the director? Participants answered using a scale from 1 (not at all) to 10 (very well). The participants also answered two more questions: (4) Did you make an effort to understand the intentions and emotions of the characters? and (5) Did you make an effort to understand the intentions of the director? The participants answered using a scale from 1 (definitely no) to 10 (definitely yes).

\subsubsection{Procedure}

The participant watched the first film, made two aesthetic judgements about it (rated that first film for 'liking' and 'wanting'), watched the second film, rated the second film for 'liking' and 'wanting', etc. up to film eight (see Note 1). Afterwards, the participant rated whether s/he understood emotions and intentions and how much s/he tried to understand them in film one and repeated those ratings for films two to eight. The intention and emotion-understanding questions were asked after participants evaluated films aesthetically, in order to avoid the possible impact of these questions on aesthetic appreciation. After the study, the participants were debriefed and reported that they had not watched the feature films prior to the study.

The study was performed in a laboratory room in daylight. The films were presented on a notebook screen with a window of $18 \times 31 \mathrm{~cm}$ and participants wore headphones. The auditory format was 24 bits, $48.000 \mathrm{~Hz}(60 \%$ of 100). The resolution of the display (generic PnP monitor) was set to the maximum possible, $1920 \times 1080$ pixels.

\subsubsection{Analysis}

We tested the hypothesis about the link between aesthetic appreciation and intention/emotion understanding using linear mixed-effects models. The analysis was conducted in R 4.0.2 (R Core Team, 2013) using the lme4 package 
for mixed-effect regression models (Bates et al., 2014). Three items about understanding the characters' intention and emotion and the director's intention were collapsed into one scale (intention/emotion understanding). This procedure was performed because theoretically in this study there is no reason to distinguish between these items. The reliability of the scale (Cronbach's $\alpha$ ) was 0.819 . Two items about the effort to understand the intentions/emotions of characters and director were collapsed into one scale (effort to understand; Cronbach's $\alpha=0.736)$. Liking and wanting judgements were collapsed into one scale as well (aesthetic appreciation; Cronbach's $\alpha=0.878$ ).

\subsection{Results}

To test the hypothesis that the aesthetic evaluation of films is related to theory of mind, a linear mixed-effects model calculated how intention/emotion understanding and effort related to it explain aesthetic appreciation (see Table 1).

Both variables were significantly and positively related to aesthetic appreciation and explained $21 \%$ of the variance (marginal $R^{2}=0.215$ ). The effect size was medium $\left(f^{2}=0.274\right)$. Local effect sizes were also calculated for emotion/intention understanding $\left(f^{2}=0.268\right)$ and effort to understand $\left(f^{2}=0.092\right)$.

\subsection{Discussion}

Study 1 aimed to investigate a link between intention/emotion understanding and aesthetic appreciation of cinema. It showed that intention/emotion understanding by self-report is positively related to aesthetic appreciation, in both their 'liking' and 'wanting' aspects. In particular, the more people think they understand the feelings and intentions of the cinema characters, the more they aesthetically enjoy that film, and vice versa.

Our second finding from this study is that it is not only the subjective understanding of a character's emotion but the effort made for this understanding that matters for aesthetic appreciation. If the character's emotions are easily

Table 1.

Regression models explaining aesthetic appreciation.

Aesthetic appreciation

\begin{tabular}{lcccccc}
\hline Theory of mind & Estimate & SE & $t$-value & $p$-value & Lower 2.5 & Upper 97.5 \\
\hline $\begin{array}{l}\text { Emotion/intention } \\
\text { understanding }\end{array}$ & 0.540 & 0.075 & 7.155 & 0.0001 & 0.392 & 0.692 \\
\begin{tabular}{l} 
Effort to understand \\
\hline
\end{tabular} & 0.309 & 0.082 & 3.780 & 0.0005 & 0.147 & 0.471 \\
\hline
\end{tabular}

Full equation: Aesthetic appreciation $=0.768+0.54 *$ Emotion/intention understanding +0.309 Effort to understand. 
understood, it is possible that the emotion-understanding process is not intensified.

Finally, we found that subjective understanding of the director's intention is significantly related to aesthetic appreciation of the film. This fact supports the idea that in perceiving the artwork, the viewer attempts to understand not only the mind of the characters, but also the mind of the one who created them. It also supports the empirical findings of Margulis and colleagues (2017), that information about the artists' intentions enhances the aesthetic appreciation of an artwork.

\section{Study 2}

In Study 1 we found that subjective understanding of the emotions/intentions of characters and the director and the effort involved in this understanding are related to the aesthetic appreciation of cinema. Study 2 was conducted to test whether there is any causation between an attempt to understand emotion and aesthetic appreciation. Participants evaluated aesthetically different movie shots either in terms of understanding emotions (experimental), or of understanding age (control). The experiment consisted of two parts, an emotion rating part and an age rating part. All participants completed both parts, and the order was counterbalanced within participants. It was expected that the attempt at understanding emotion has an effect on aesthetic appreciation. That is, when participants attempt to understand the emotions of the characters (as opposed to age understanding), they evaluate movie shots as more aesthetically appealing.

\subsection{Method}

\subsubsection{Participants}

Based on Study 1, the effect of subjective emotion/intention understanding on aesthetic appreciation was medium $\left(f^{2}=0.268\right)$. Power analysis, conducted in G*Power (Faul et al., 2007), indicated that a minimal sample size of 40 people was necessary to test the differences between two dependent groups with a medium effect size and power $=0.95$. There were two rounds of data collection with several months inbetween. Twenty-seven participants were initially recruited at university campus. Two of them understood the hypothesis of the study and were excluded. During the second round of data collection eighteen participants were recruited. Forty-three participants were included in the final sample. Their age ranged from 19 to 38 years $\left(M_{\text {age }}=22.14, \mathrm{SD}=3.61\right.$; 16 males). Informed consent was obtained, and the experiment was carried out in accordance with the Code of Ethics of the World Medical Association 
(Declaration of Helsinki). After the study, the participants were debriefed and they reported that they had not viewed the photos before the study.

\subsubsection{Materials}

Fifty coloured photos were collected from different cinema movies and from a professional art photograph collection assembled for this experiment. We attempted to collect photos which depict one main character and avoided photos where the emotional state of the character was very clear (such as images of smiling or crying people). The size of all photos was $1081 \times 747$ pixels; $12 \times$ $17.5 \mathrm{~cm}$. The display characteristics were the same as in Study 1. The experiment was built and presented in PsychoPy (Peirce, 2007, 2009).

\subsubsection{Procedure}

Participants were instructed that they would be presented with an artistic photo, extracted from a film and representing a character from that film. Their task was to evaluate either the emotional state of the character or the character's age. In the first case, the word 'EMOTION' was displayed above the photo (see Fig. 1, top row). On the left and on the right, two answers were displayed (for example, 'Dreamy' or 'Sad'). The set included negative emotional states (e.g., miserable, lonely, desperate, depressive) as well as positive ones (e.g., dreamy, happy, hopeful, grateful). Participants chose between the emotions of the same valence. The participant was instructed to choose the emotion that he/she thinks is correct using a scale from 1 to 4 (1, definitely emotion on the left; 2 , probably emotion on the left; 3 , probably emotion on the right; 4 , definitely emotion on the right). In the second case, the word 'AGE' was displayed above the photo (see Fig. 1, bottom row). On the left and on the right, two answers were displayed (for example, ' 25 ' or ' 30 '). The participant must choose the age that he/she thinks is correct using a scale from 1 to 4 . The ageunderstanding condition was chosen as a control condition, for comparison with the experimental emotion-understanding condition (because understanding the age of a character does not imply understanding the character's mental state). Also, the age condition was chosen because age judgements are complex and require effort, thus they can be comparable to emotion-understanding judgements. For instance, age judgements are used in neuroimaging studies as control condition for mentalizing tasks (Bzdok et al., 2012; Eddy et al., 2017; Nolte et al., 2013).

Right after making a decision about the character's age or emotional state (depending on the condition), the participant was asked to rate the photo aesthetically, i.e., to make aesthetic 'liking' and 'wanting' judgements about the photo. In this study, to evaluate aesthetic 'liking' judgements we asked our participants how much a photo made an impact on them from an aesthetic point of view, instead of asking whether the photo was aesthetically appealing 

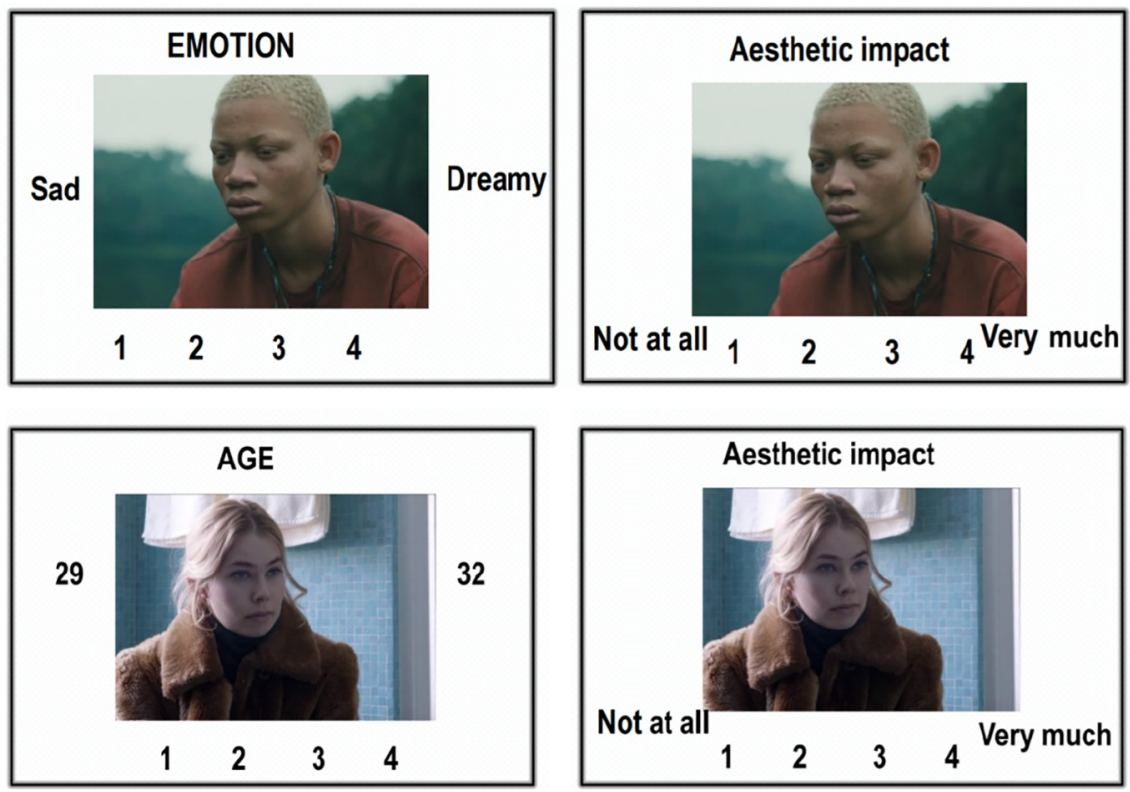

Figure 1. Emotion-understanding condition of the task (top row) and age-understanding condition of the task (bottom row).

(“How much did this photo make an aesthetic impact on you?"). Participants answered on a scale from 1 (not at all) to 4 (very much). To evaluate aesthetic 'wanting' judgements, we asked the participants how likely they would be to go to the cinema to watch the whole film. The participants answered using a scale from 1 (definitely not) to 4 (definitely yes). The time was not limited.

After the participant answered the question about the character (either about the age or about the emotional state) and evaluated the photo aesthetically, a fixation cross appeared for a duration of $1 \mathrm{~s}$. After the fixation cross, the next photo was presented. All photos were presented in a random order. Half of the photos (25 randomly assigned photos) were presented in the emotion-understanding condition, and the other half were presented in the ageunderstanding condition (see Fig. 1 top and bottom rows). The conditions and photos were balanced among the participants.

Prior to the main experiment of 50 trials ( 25 trials in the age-understanding condition, 25 trials in the emotion-understanding condition), the participants had an opportunity to practice performing a short training task. A withinpersons design was used in this study.

\subsubsection{Analysis}

To test the hypothesis that the attempt at emotion understanding has a positive impact on aesthetic appreciation, we compared the mean aesthetic evaluation 
of images in the emotion-understanding condition and the age-understanding condition using the Wilcoxon signed-rank test. Nonparametric statistic was used because the data were not normally distributed (Shapiro-Wilk test, $p$ value $<0.05)$. As in Study 1, 'Liking' and 'Wanting' items were collapsed into one scale of aesthetic appreciation (Cronbach's $\alpha=0.863$ ).

\subsection{Results}

The difference in aesthetic appreciation in the emotion-understanding condition $v s$ the age-understanding condition was significant with medium effect size $(Z=-2.920, p=0.003, r=0.315)$. The images were more aesthetically appealing in the emotion-understanding condition (Median $=2.54$, $\mathrm{IQR}=0.50)$ compared to the age-understanding condition (Median $=2.38$, $\mathrm{IQR}=0.44)$.

\subsubsection{Additional Data Analysis}

Firstly, we analyzed the degree to which the age and emotion responses were variable. If there was $100 \%$ agreement on the age judgements for most of the pictures in contrast to lots of disagreement on the emotion judgements, it might imply that the age judgements were a lot easier. A $\chi^{2}$ test of the emotion judgements was significant $(p<0.05)$ for 13 images out of 50, while for the age judgements it was significant for nine images out of 50. This does not imply that the age judgements were easier compared to emotion judgements.

To assess the effect of confidence ratings of both age and emotion judgements, we built a multiple linear regression model. Confidence was entered as independent variable. Responses "definitely emotion/age on the left/right" were coded as high-confidence responses, while responses "probably emotion/ age on the left/right", as low-confidence responses. There was no significant effect of confidence on aesthetic appreciation in the age-understanding condition $(p=0.849)$. To the contrary, confidence increased aesthetic appreciation in the emotion-understanding condition $(b=0.18, \mathrm{SE}=0.06, p=0.003)$.

\subsection{Discussion}

The aim of Study 2 was to investigate whether there is causation between an attempt to understand emotion of a character and aesthetic evaluation of a photo. It was found that evaluation of mental states elicits a higher aesthetic appreciation of photos, as opposed to evaluation of formal traits (age).

\section{Study 3}

The correctness of emotion/intention understanding was not assessed in Studies 1 and 2. Study 3 was conducted to assess that correctness and test the hypothesis that better understanding of another's emotions is related to greater 
aesthetic appreciation. That is, when participants correctly identify the emotion of the movie character, they aesthetically appreciate the movie more. Study 3 was also conducted to replicate the findings of Study 2. That is, that attempting to understand the emotions and intentions of the characters (as opposed to attempt to understand age) has a positive impact on the aesthetic appreciation of movie shots.

\subsection{Methods}

\subsubsection{Participants}

Based on Study 2, the effect of emotion/intention understanding on aesthetic appreciation was medium $(r=0.315)$. Power analysis, conducted in $\mathrm{G}^{*}$ Power (Faul et al., 2007) indicated that a minimal sample size of 35 people was necessary to test the differences between two dependent groups with a medium effect size and power $=0.95$. Forty participants took part in the study; their age ranged from 20 to 22 years $\left(M_{\mathrm{age}}=20.46, \mathrm{SD}=0.68\right.$; seven males). Informed consent was obtained, and the experiment was carried out in accordance with the Code of Ethics of the World Medical Association (Declaration of Helsinki). After the study, the participants were debriefed and asked whether they had seen the films before the study.

\subsubsection{Materials and Procedure}

The procedure of Study 3 was similar to that in Study 2. The only difference was in the set of images. Twenty-six images were displayed in the emotion-understanding condition, while twenty-six different images were displayed in the age-understanding condition. Overall, there were 52 trials in the experiment.

A different set of images was used in this study. Fifty-two images were extracted from different films (all film directors are highly professionally recognized and have received prestigious awards at film festivals). All images were close-ups, portraying only one character. Because in this study we calculated the correctness of emotion understanding, we avoided images with ambivalent and neutral emotional expressions. To calculate the correctness of emotion understanding, the consensus approach was used: ten subjects (not included in the main sample) previously evaluated the experienced emotion; if at least six of them considered that the character experienced the emotion, it was considered as a correct response. No descriptors of possible mental states were given. A consensus approach was preferred to a realistic approach and a pragmatic approach, because these two cannot be easily applied in art, as opposed to real-life communication. Based on the consensus approach, the correctness of emotion understanding was calculated as follows: (1) the correct response (expressed on a scale from 1 to 4 ) was subtracted from the participant's actual 
response; (2) the obtained result was inversed. For example, if the correct response was 4 and the participant's response was 4 , the incorrectness of understanding was 0 and the correctness $=4$. Finally, the correctness of age understanding was not assessed.

We attempted to reach uniformity of stimuli in the emotion-understanding condition and the age-understanding condition. There was an equal number of black-and-white and coloured images, as well as images with positive/negative emotions across these two conditions. Because the familiarity with the films could affect the understanding of emotion and the aesthetic appreciation, we attempted to choose images without famous actors or other easily recognizable treats. Four of them reported they recognized the image from "The Passion of Joan of Arc" (by Carl Theodor Dreyer); five participants reported they recognized the image from "Psycho" (by Alfred Hitchcock); three participants reported they recognized the image from "Loveless" (by Andrey Zvyagintsev). No participants recognized more than two images out of 52. Because of the small number of recognized images, no data were excluded from the analysis.

\subsection{Results}

As for Studies 1 and 2, 'liking' and 'wanting' judgements were collapsed in one aesthetic judgement scale (Cronbach's $\alpha=0.853$ ). Nonparametric statistics was used because the data were not normally distributed (Shapiro-Wilk test, $p$ value $<0.05)$. The Wilcoxon signed-ranks test showed that the difference in aesthetic appreciation in the emotion-understanding condition vs the ageunderstanding condition was significant with medium effect size $(Z=-4.083$, $p=0.0001, r=0.457)$. Images were considered as more aesthetically appealing in the emotion-understanding condition $($ Median $=2.49, \mathrm{IQR}=0.481) v \mathrm{~s}$ the age-understanding condition (Median $=2.29, \mathrm{IQR}=0.625$ ).

A linear mixed-effects model calculated how emotion understanding explains aesthetic appreciation (see Table 2). Emotion understanding was significantly and positively related to aesthetic appreciation with a small effect size $\left(f^{2}=0.02\right)$. On average, the participants managed to guess correctly the emotions of the characters $(M=3.211, \mathrm{SD}=0.253)$.

\subsubsection{Additional Analysis}

To assess the effect of confidence ratings of both age and emotion judgements, we built a multiple linear regression model. Confidence was entered as independent variable. Responses "definitely emotion/age on the left/right" were coded as high-confidence responses, while responses "probably emotion/age on the left/right" were as low-confidence responses. There was no significant effect of confidence on aesthetic appreciation in the age-understanding condi- 
Table 2.

Regression model explaining aesthetic appreciation.

Aesthetic appreciation

\begin{tabular}{lllcccc}
\hline Variable & Estimate & SE & $t$-value & $p$-value & Lower 2.5 & Upper 97.5 \\
\hline $\begin{array}{l}\text { Correct } \\
\text { understanding }\end{array}$ & 0.081 & 0.030 & 2.683 & 0.012 & 0.021 & 0.140 \\
\hline
\end{tabular}

$\mathrm{R}^{2}=0.02$. Full equation: Aesthetic appreciation $=2.24+0.08 *$ Correct understanding

tion $(p=0.136)$. To the contrary, confidence increased aesthetic appreciation in the emotion-understanding condition $(b=0.28, \mathrm{SE}=0.06, p<0.0001)$.

\subsection{Discussion}

The aim of Study 3 was to get a better understanding of the direction of the relationships between the correctness of emotion understanding and aesthetic appreciation. It was found that better emotion understanding is positively related to aesthetic appeal of the images. However, the effect size of this causation was small. Previous studies showed that the link between social cognition and perception of fiction has a small effect size (Dodell-Feder and Tamir, 2018).

Study 3 also replicated the findings of Study 2 and showed that an attempt to evaluate the minds of others has a positive impact on aesthetic appreciation, as opposed to attempts to understand the formal traits (age).

\section{Study 4}

Earlier it was found that more extensive elaboration increases aesthetic appreciation (Belke et al., 2015). Study 4 was conducted to ensure that the findings of Studies 2 and 3 are not caused by differences in cognitive engagement between experimental and control conditions (emotion understanding vs age understanding). In Study 4, in the experimental condition participants were asked to write down (rather than choose between two options) the mental state of the character. In the control condition participants were asked to pick a colour which resembles best the background of the movie shot.

\subsection{Method}

\subsubsection{Participants}

Based on Study 2, the effect of emotion/intention understanding on aesthetic appreciation was medium $(r=0.315)$. Power analysis, conducted in $\mathrm{G}^{*}$ Power (Faul et al., 2007) indicated that a minimal sample size of 35 people was necessary to test the differences between two dependent groups with a medium 
effect size and power $=0.95$. Data were collected online on the platform Yandex Toloka (https://toloka.yandex.com/). Participants received a remuneration for their participation. Forty participants were recruited. One of them did not complete more than $30 \%$ of the study and was excluded. The final sample included 39 participants (20 females; age $18-62$ years, $M=33.56, \mathrm{SD}=10.74$ ). Informed consent was obtained, and the experiment was carried out in accordance with the Code of Ethics of the World Medical Association (Declaration of Helsinki).

\subsubsection{Procedure}

The same set of images as in Study 3 was used in Study 4. The experiment was built and presented in PsychoPy (Peirce, 2007, 2009) on the Pavlovia platform (https://pavlovia.org/). Participants were presented with a movie shot. In the experimental condition, they were asked "What is this character feeling?" and asked to write down the answer in one or two words. The movie shot was presented for $3 \mathrm{~s}$ only, but the response was not limited in time. In the control condition, participants were asked to pick a colour which most accurately resembled the movie shot's background. Colours were presented on the screen under the movie shot. There were 50 colours: 25 monochromatic colours from black to white, varying in luminosity, and 25 colours from red to purple, varying in hue.

As in Studies 2 and 3, after answering questions about the colour of the movie shot or the feelings of the character (depending on the condition), the participant was asked to rate the photo aesthetically on a scale from 1 to 4 . They were asked to rate the liking of the photo on a scale from 1 to 4 and the interest elicited by the photo ( 1 - not interesting, 4 - interesting) (Note 2). Time was not limited. Prior to the main experiment of 50 trials ( 25 trials in the experimental condition, 25 in the control condition), the participants had an opportunity to practice performing a short training task. Aesthetic evaluation of the movie shots was not included in this practice trial.

After participants answered the question about the movie shot (either about the colour or about the mental state) and evaluated the photo aesthetically, a fixation cross appeared for a duration of $1 \mathrm{~s}$. After the fixation cross, the next photo was presented. All photos were presented in a random order. Half of the photos (25 randomly assigned photos) were presented in the experimental condition, and the other half were presented in the control condition. The conditions and photos were balanced among the participants. A within-persons design was used in this study.

\subsection{Results}

To test the hypothesis that an attempt to understand the emotion has a positive impact on aesthetic appreciation, we compared the mean aesthetic evaluation of images in the emotion-understanding condition and the control condition. 
Parametric statistics was used because the data were normally distributed (Shapiro-Wilk test, $p$ value $>0.05$ ). As in the previous studies, 'liking' and 'interest' items were collapsed in one scale of aesthetic appreciation because they correlated highly $(r=0.95)$.

The difference in aesthetic appreciation in the emotion-understanding condition $v s$ the colour attribution condition was significant with medium effect size $\left(t_{38}=-3.61, p=0.001, d=0.573\right)$. The movie shots were more aesthetically appealing in the emotion-understanding condition $(M=2.81, \mathrm{SD}=0.60)$ compared to the control condition $(M=2.65, \mathrm{SD}=0.59)$.

\subsection{Discussion}

The aim of Study 4 was to test if the emotion-understanding effect on aesthetic appreciation is significant with a different control condition - the colour attribution task. This study confirmed that the evaluation of mental states elicits higher aesthetic appreciation of movie shots, as opposed to the evaluation of formal traits of these shots (colour).

\section{General Discussion}

The aim of this research was to investigate the relationship between emotion/ intention understanding and aesthetic appreciation. The results of Study 1 showed that there is a link between subjective intention/emotion understanding and the aesthetic appreciation of cinema. The more the viewers think that they understand the feelings/intentions of the characters and the artist, and the more effort they make to understand them, the more they aesthetically appreciate the cinema and vice versa. Understanding the artist's intentions can be considered a more complicated cognitive process, because the artist is not directly represented on the screen, as the characters are. However, our result supposes that when perceiving a film, the viewer attempts to understand the artist's intentions, and the subjective understanding of these intentions correlates positively with aesthetic appreciation.

Study 2 was conducted to investigate whether there is not only a correlation but a causation between engaging in emotion understanding and aesthetic appreciation of visual art. The study confirmed that the attempt to understand a character's emotional experience as opposed to their formal traits (such as age) influences the viewer's aesthetic appreciation of photos with medium effect size. This result is in line with the findings of Margulis and colleagues (2017) who found that information about the mind of the other positively affects the aesthetic appreciation of poetry and music. Our result supports their findings in visual art. However, it is not clear whether the mechanisms that connect understanding the mind of another and appreciation of music, poetry and visual arts are the same. 
It should be mentioned that relationship between theory of mind and aesthetic appreciation might be bidirectional, which is particularly relevant to the findings of correlational Study 1. This might indicate that there are two different mechanisms which link mind reading and aesthetic appreciation. Firstly, better understanding emotion increases aesthetic appreciation of the artwork, which might signify a cognitively-induced gratification mechanism. Mind reading helps us to make sense of complex and contradictive stimuli, and this can be one reason why it increases aesthetic appeal. This mechanism can be related to the efficient processing theory of aesthetics which assumes that efficiently processed stimuli are the most aesthetically appealing (Reber et al. 2004; Renoult, 2016). Secondly, higher enjoyment of an artwork might lead to increased efforts to derive a correct understanding of emotion, signifying the presence of a processing-motivating mechanism.

The second major finding of this study is that the correctness of emotion understanding is related to enhanced aesthetic appreciations. That is, the better the viewer understands the emotions of the characters, the higher is his/her aesthetic appreciation. This result supports the idea that along with experiencing emotions during aesthetic cognition, understanding them is important for aesthetic appreciation. That is, it is not only the mirroring of the emotional state of the characters that impacts aesthetic appreciation (as some early aesthetic theories have claimed; e.g., Aristotle's idea of mimesis and catharsis), but cognitive appraisal of these emotions plays a role as well. It should be mentioned that the effect was found although the emotional states of the characters were not always designated as positive.

Evaluation of the mental state of the others may enhance the aesthetic appreciation for several reasons. Firstly, it is possible that there is direct link between the attempt to understand the mental states of others and aesthetic appreciation. Secondly, it possible that some other variables, such as empathy and self-identification, mediate the link between mentalizing and aesthetic appreciation. That is, understanding the mental states of others might facilitate empathy and self-identification, which in turn enhance aesthetic appreciation (Freedberg and Gallese, 2007; Igartua, 2010).

There are some important limitations in this research. In Study 1, emotion/ intention understanding and the effort made for this understanding were measured using self-report. The self-report captures explicit and reflexive processes, but not implicit automatic processes, which are also important in intention/emotion understanding. Future studies may use more objective methods to measure the intensity of intention/emotion-understanding processes, such as neuroimaging.

Overall, the research showed that evaluating the minds of others and emotion understanding play a role in the aesthetic appreciation of visual art. Some suggestions for future studies can be derived. We used different types of visual 
media across studies: moving images in Study 1, artistic photos in Study 2, movie-stills in Studies 3 and 4. This set of stimuli consists of different types of sensorial information. Given the we found similar effects across studies it is possible that the mind-reading effect generalizes across various visual-media formats. It is possible that a higher-order cognitive mechanism underlies this effect and operates above the sensorial input it receives. Future studies may investigate this mechanism and whether intention and emotion understanding have an impact on other types of art, such as literature, abstract art and classical art. Most importantly, researchers should investigate whether the mechanisms of interactions between emotion understanding and aesthetic appreciation are similar in different art forms or not. Finally, although emotion understanding plays a role in aesthetic evaluation, it is not the only factor that predicts it. It is thus important to investigate how emotion understanding interacts with other psychological variables known to impact aesthetic judgement (for example, self-related interpretation).

\section{Acknowledgement}

The author is thankful to Igor Vlasov for his valuable comments and advise.

\section{Data Availability Statement}

Data directly supporting the publication can be found at https://osf.io/35k2d/

\section{Conflicts of Interest}

The author declares to have no conflicts of interest regarding the present work.

\section{Notes}

1. Both 'wanting' and 'liking' judgements were included in line with Chatterjee's neuroaesthetical model that designates two types of reward brought about by two distinct brain systems (Chatterjee, 2011).

2. 'Liking' and 'interest' judgements were included based on Berlyne's differentiation between interestingness and pleasingness as two dimensions of aesthetic response (Berlyne, 1974).

\section{References}

Baron-Cohen, S., Wheelwright, S., Hill, J., Raste, Y. and Plumb, I. (2001). The "Reading the Mind in the Eyes" Test revised version: a study with normal adults, and adults with Asperger syndrome or high-functioning autism, J Child Psychol. Psychiatry 42, 241-251. doi: 10.1017/S0021963001006643.

Bates, D., Mächler, M., Bolker, B. and Walker, S. (2014). Fitting Linear Mixed-Effects Models using lme4. ArXiv:1406.5823 [Stat]. Retrieved from http://arxiv.org/abs/1406.5823. 
Belke, B., Leder, H., Carbon, C.C. (2015) When challenging art gets liked: evidences for a dual preference formation process for fluent and non-fluent portraits, PLoS ONE 10, e0131796. doi: 10.1371/journal.pone.0131796.

Berlyne, D. E. (1974). Studies in the New Experimental Aesthetics: Steps toward an Objective Psychology of Aesthetic Appreciation. Hemisphere, Oxford, UK.

Black, J. E. and Barnes, J. L. (2015a). The effects of reading material on social and non-social cognition, Poetics 52, 32-43. doi: 10.1016/j.poetic.2015.07.001.

Black, J. and Barnes, J. L. (2015b). Fiction and social cognition: The effect of viewing awardwinning television dramas on theory of mind, Psychol. Aesthet. Creat. Arts 9, 423-429. doi: 10.1037/aca0000031.

Bullot, N. J. and Reber, R. (2013). The artful mind meets art history: Toward a psycho-historical framework for the science of art appreciation,. Behav. Brain Sci. 36, 123-137. doi: 10.1017/ S0140525x12000489.

Bzdok, D., Langner, R., Hoffstaedter, F., Turetsky, B. I., Zilles, K. and Eickhoff, S. B. (2012). The modular neuroarchitecture of social judgments on faces, Cereb Cortex 22, 951-961. doi: 10.1093/cercor/bhr166.

Charnes, L. (2007). "Hamlet" Without Hamlet (review). Shakespeare Q. 58, 538-542. doi: 10.1353/shq.2007.0054.

Chatterjee, A. (2011). Neuroaesthetics: a coming of age story, J. Cogn. Neuriosci. 23, 53-62. doi: 10.1162/jocn.2010.21457.

Costanzo, M. and Archer, D. (1989). Interperting the expressive behavior of others: The Interpersonal Perception Task, J. Nonverbal Behav. 13, 225-245. doi: 10.1007/BF00990295.

Dodell-Feder, D. and Tamir, D. I. (2018). Fiction reading has a small positive impact on social cognition: A meta-analysis, J. Exp. Psychol. Gen. 147, 1713-1727.doi: 10.1037/xge0000395.

Eddy, C. M., Cavanna, A. E. and Hansen, P. C. (2017). Empathy and aversion: the neural signature of mentalizing in Tourette syndrome, Psychol. Med. 47, 507-517. doi: 10.1017/ S0033291716002725.

Faul, F., Erdfelder, E., Lang, A.-G. and Buchner, A. (2007). G*Power 3: A flexible statistical power analysis program for the social, behavioral, and biomedical sciences, Behav. Res. Meth. 39, 175-191. doi: 10.3758/BF03193146.

Flavell, J. H. (2004). Development of knowledge about vision, in: Thinking and seeing: Visual metacognition in adults and children, D. T. Levin (Ed.), pp. 13-36. MIT Press, Cambridge, MA, USA.

Freedberg, D. and Gallese, V. (2007). Motion, emotion and empathy in esthetic experience, Trends Cogn. Sci. 11, 197-203. doi: 10.1016/j.tics.2007.02.003.

Gallagher, H. L. and Frith, C. D. (2003). Functional imaging of 'theory of mind', Trends Cogn. Sci. 7, 77-83. doi: 10.1016/s1364-6613(02)00025-6.

Höfel, L., \& Jacobsen, T. (2007). Electrophysiological indices of processing aesthetics: Spontaneous or intentional processes? Int. J. Psychophysiol. 65, 20-31. doi: 10.1016/j. ijpsycho.2007.02.007.

Igartua, J.-J. (2010). Identification with characters and narrative persuasion through fictional feature films, Communications 35, 347-373. doi: 10.1515/comm.2010.019.

Juslin, P. N. and Laukka, P. (2003). Communication of emotions in vocal expression and music performance: different channels, same code? Psychol. Bull. 129, 770-814. doi: 10.1037/ 0033-2909.129.5.770. 
Kidd, D. C. and Castano, E. (2013). Reading literary fiction improves theory of mind, Science 342, 377-380. doi: 10.1126/science.1239918.

Kirk, U., Skov, M., Hulme, O., Christensen, M. S. and Zeki, S. (2009). Modulation of aesthetic value by semantic context: an fMRI study, NeuroImage 44, 1125-1132. doi: 10.1016/j. neuroimage.2008.10.009.

Leder, H., Belke, B., Oeberst, A. and Augustin, D. (2004). A model of aesthetic appreciation and aesthetic judgments, Br. J. Psychol. 95, 489-508. doi: 10.1348/0007126042369811.

Levin, R. C. (1994). More nuns and nunneries and Hamlet's speech to Ophelia, Notes Queries $41,41-42$.

Levin, D. T., Hymel, A. M. and Baker, L. (2013). Belief, desire, action, and other stuff: Theory of mind in movies, in: Psychocinematics: Exploring Cognition at the Movies, A. P. Shimamura (Ed.), pp. 244-266. Oxford University Press, Oxford, UK. doi: 10.1093/acprof:o so/9780199862139.003.0013.

Mar, R. A., Oatley, K., Hirsh, J., dela Paz, J. and Peterson, J. B. (2006). Bookworms versus nerds: Exposure to fiction versus non-fiction, divergent associations with social ability, and the simulation of fictional social worlds, J. Res. Personal. 40, 694-712. doi: 10.1016/j. jrp.2005.08.002.

Mar, R. A., Oatley, K. and Peterson, J. B. (2009). Exploring the link between reading fiction and empathy: Ruling out individual differences and examining outcomes, Communications 34, 407-428. doi: 10.1515/COMM.2009.025.

Margulis, E. H., Levine, W. H., Simchy-Gross, R. and Kroger, C. (2017). Expressive intent, ambiguity, and aesthetic experiences of music and poetry, PLOS ONE 12, e0179145. doi: 10.1371/journal.pone.0179145.

Marković, S. (2012). Components of aesthetic experience: aesthetic fascination, aesthetic appraisal, and aesthetic emotion, i-Perception 3, 1-17. doi: 10.1068/i0450aap.

Mumper, M. L. and Gerrig, R. J. (2017). Leisure reading and social cognition: A meta-analysis, Psychol. Aesthet. Creat. Arts 11, 109-120. doi: 10.1037/aca0000089.

Nolte, T., Bolling, D. Z., Hudac, C. M., Fonagy, P., Mayes, L. and Pelphrey, K. A. (2013). Brain mechanisms underlying the impact of attachment-related stress on social cognition, Front. Hum. Neurosci. 7, 816. doi: 10.3389/fnhum.2013.00816

Panero, M. E., Weisberg, D. S., Black, J., Goldstein, T. R., Barnes, J. L., Brownell, H. and Winner, E. (2016). Does reading a single passage of literary fiction really improve theory of mind? An attempt at replication, J. Pers. Soc. Psychol. 111, e46-e54. doi: 10.1037/ pspa0000064

Parsons, M. J. (1987). How We Understand Art: A Cognitive Developmental Account of Aesthetic Experience. Cambridge University Press, New York, NY, USA.

Peirce, J. W. (2007). PsychoPy — Psychophysics software in Python, J. Neurosci. Meth. 162, 8-13. doi: 10.1016/j.jneumeth.2006.11.017.

Peirce, J. W. (2009). Generating stimuli for neuroscience using PsychoPy, Front. Neuroinform. 2, 10. doi: 10.3389/neuro.11.010.2008.

Pelowski, M. and Akiba, F. (2011). A model of art perception, evaluation and emotion in transformative aesthetic experience, New Ideas Psychol. 29, 80-97. doi: 10.1016/j. newideapsych.2010.04.001.

Pignocchi, A. (2015). Pourquoi Aime-t-on un Film? Quand les Sciences Cognitives Discutent des Goûts et des Couleurs. Odile Jacob, Paris, France. 
R Core Team (2013). R: A Language and Environment for Statistical Computing. R Foundation for Statistical Computing, Vienna, Austria. URL http://www.R-project.org/.

Reber, R., Schwarz, N. and Winkielman, P. (2004). Processing fluency and aesthetic pleasure: is beauty in the perceiver's processing experience? Pers. Soc. Psychol. Rev. 8, 364-382. doi: 10.1207/s15327957pspr0804_3.

Renoult, J.P. (2016). The evolution of aesthetics: a review of models, in: Aesthetics and Neuroscience Scientific and Artistic Perspectives, Z. Kapoula and M. Vernet (Eds), pp. 271-299, Springer International Publishing, Cham, Switzerland. doi: 10.1007/978-3-319-46233$2 \_17$.

Werstine, P. (1988). The textual mystery of Hamlet, Shakespeare Q. 39, 1-26. doi: $10.2307 / 2870584$.

Weth, K., Raab, M. H. and Carbon, C.-C. (2015). Investigating emotional responses to self-selected sad music via self-report and automated facial analysis, Mus. Sci. 19, 412-432. doi: $10.1177 / 1029864915606796$.

Wolz, S. H. and Carbon, C.-C. (2014). What's wrong with an art fake? Cognitive and emotional variables influenced by authenticity status of artworks, Leonardo 47, 467-473. doi: 10.1162/ LEON_a_00869.

Zunshine, L. (2003). Theory of mind and experimental representations of fictional consciousness, Narrative 11, 270-291. 